\title{
ASYMPTOTIC EXPANSION FOR DAMPED WAVE EQUATIONS WITH PERIODIC COEFFICIENTS
}

\author{
R. ORIVE and E. ZUAZUA \\ Departamento de Matemática Aplicada, Facultad de Ciencias Matemáticas \\ Universidad Complutense de Madrid, 28040 Madrid, EspañA. \\ orive@sunma4.mat.ucm.es, zuazua@eucmax.sim.ucm.es \\ A. F. PAZOTO \\ Departamento de Métodos Matemáticos, Instituto de Matemática \\ Universidade Federal do Rio de Janeiro, P.O.Box 68530, 21945-979 Rio de Janeiro, BrasiL. \\ ademir@acd.ufrj.br
}

\begin{abstract}
We consider a linear dissipative wave equation in $\boldsymbol{R}^{N}$ with periodic coefficients. By means of Bloch wave decomposition, we obtain an expansion of solutions as $t \rightarrow \infty$ and conclude that, in a first approximation, the solutions behave as the homogenized heat kernel.
\end{abstract}

\section{Introduction}

\subsection{Setting of the problem}

This paper is concerned with the analysis of the asymptotic behavior, as $t \rightarrow \infty$, of the solutions of

$$
\left\{\begin{array}{l}
\rho(x) u_{t t}-\frac{\partial}{\partial x_{k}}\left(a_{k \ell}(x) \frac{\partial u}{\partial x_{\ell}}\right)+a_{0} \rho(x) u_{t}=0 \quad \text { in } \mathbb{R}^{N} \times(0, \infty) \\
u(x, 0)=\varphi^{0}(x) \\
u_{t}(x, 0)=\varphi^{1}(x)
\end{array}\right.
$$

where the coefficients satisfy

$$
\left\{\begin{array}{l}
\left.a_{k \ell} \in L_{\#}^{\infty}(Y) \text { where } Y=\right] 0,2 \pi\left[^{N} \text {, i.e., each } a_{k \ell}\right. \text { is a } \\
Y \text {-periodic bounded measurable function defined on } \boldsymbol{R}^{N}, \\
\exists \alpha>0 \text { such that } a_{k \ell}(x) \eta_{k} \eta_{\ell} \geq \alpha|\eta|^{2} \quad \forall \eta \in \mathbb{R}^{N}, \text { a.e. } x \in \mathbb{R}^{N}, \\
a_{k \ell}=a_{\ell k} \quad \forall k, \ell=1, \ldots, N, \text { and } \\
a_{0} \text { is a positive constant, }
\end{array}\right.
$$

and

$$
\left\{\begin{array}{l}
\rho \in L_{\#}^{\infty}(Y) \text {, i.e., } \rho \text { is } Y \text {-periodic, and } \\
\exists \rho_{0}, \rho_{1} \in \mathbb{R}_{+}, \text {such that } 0<\rho_{0} \leq \rho(x) \leq \rho_{1} \text {, a.e. } x \in Y .
\end{array}\right.
$$


The equation (1.1) has a dissipative nature. Indeed, the energy associated to (1.1), given by

$$
E(t)=\frac{1}{2} \int_{R^{N}}\left[\rho(x)\left|u_{t}\right|^{2}+a_{k \ell}(x) \frac{\partial u}{\partial x_{\ell}} \frac{\partial u}{\partial x_{k}}\right] d x
$$

is decreasing

$$
\frac{\partial E}{\partial t}=-a_{0} \int_{R^{N}} \rho(x)\left|u_{t}\right|^{2} d x .
$$

Moreover, solutions of (1.1) satisfy the conservation law

$$
\frac{\partial}{\partial t} \int_{R^{N}}\left(u_{t}+a_{o} u\right) \rho(x) d x=\int_{R^{N}} \frac{\partial}{\partial x_{k}}\left(a_{k \ell}(x) \frac{\partial u}{\partial x_{\ell}}\right) d x=0 .
$$

That is, the mass of $u_{t}+a_{o} u$ with respect to the weight $\rho(\cdot)$ is conserved along time:

$$
m_{\rho}\left(u_{t}+a_{o} u\right)=m_{\rho}\left(\varphi^{1}+a_{0} \varphi^{0}\right)=\int_{R^{N}}\left(\varphi^{1}+a_{0} \varphi^{0}\right) \rho(x) d x .
$$

Using Bloch waves decomposition together with a choice of a convenient Lyapunov function we obtain the asymptotic expansion of the solutions of (1.1). In particular we conclude that solutions behave as the homogenized heat kernel as $t \rightarrow \infty$. In fact, equation (1.1) can be viewed as a heat equation "perturbed" by the second order term $\rho(x) u_{t t}$, that introduces oscillations that, according to our analysis, are not strong enough to change the behavior of solutions as $t \rightarrow \infty$ in a first approximation.

To be more precise, equation (1.1) can be viewed as a perturbation of the parabolic equation

$$
\left\{\begin{array}{l}
\rho(x) a_{0} u_{t}-\frac{\partial}{\partial x_{k}}\left(a_{k \ell}(x) \frac{\partial u}{\partial x_{\ell}}\right)=0 \quad \text { in } \boldsymbol{R}^{N} \times(0, \infty) \\
u(x, 0)=\varphi^{0}(x) .
\end{array}\right.
$$

The asymptotic behavior of solutions of (1.5) is well known when $\rho=1, a_{0}=1$. The first term in the asymptotic expansion was obtained in ${ }^{6}$. It was shown that

$$
t^{\frac{N}{2}\left(1-\frac{1}{p}\right)}\left\|u(t)-m(\varphi) G_{h}(\cdot, t)\right\|_{p} \longrightarrow 0, \text { as } t \rightarrow \infty, 1 \leq p \leq \infty,
$$

where

$$
m(\varphi)=\int_{R^{N}} \varphi d x
$$

and $G_{h}$ is the fundamental solution of the homogenized system

$$
\left\{\begin{array}{l}
u_{t}-q_{k \ell} \frac{\partial^{2} u}{\partial x_{\ell} x_{k}}=0 \quad \text { in } \boldsymbol{R}^{N} \times(0, \infty) \\
u(x, 0)=\delta_{0}(x) .
\end{array}\right.
$$


Here and in the sequel we denote by $\delta_{0}$ the Dirac delta at the origin and by $\left\{q_{j k}\right\}_{j, k=1}^{N}$ the homogenized coefficients associated to the periodic matrix with coefficients (1.2). The constant $m(\varphi)$ is the mass of the solution of (1.5) which is conserved along time, i.e.,

$$
\frac{\partial}{\partial t}[m(u(\cdot, t))]=0, \quad \forall t \in(0, \infty) .
$$

Later on, in J.H. Ortega and E. Zuazua ${ }^{7}$, the asymptotic expansion as $t \rightarrow \infty$ of the solutions of (1.5) with $L^{1}\left(\boldsymbol{R}^{N}\right) \cap L^{2}\left(\mathbb{R}^{N}\right)$ initial data was obtained by means of the Bloch wave decomposition (see ${ }^{3}$ and ${ }^{4}$ for an introduction to Bloch waves). Their first result was stablished in the $L^{2}\left(\mathbb{R}^{N}\right)$-setting and then, thanks to the parabolic regularizing effect, a converge result in $L^{\infty}\left(\mathbb{R}^{N}\right)$ was derived. The results in ${ }^{7}$ are, to some extent, an extension of those of ${ }^{5}$ on the constant coefficient heat equation. The analysis in ${ }^{7}$ can be easily adapted to the general parabolic equation (1.5) with variable, periodic density $\rho$.

This work is devoted to adapt the analysis in ${ }^{7}$ to the case of the dissipative wave equation (1.1) under consideration.

Our main result will only be given for the solution $u$ of (1.1) in the $L^{2}\left(\mathbb{R}^{N}\right)$ setting with $L^{2} \cap L^{1}\left(\mathbb{R}^{N}\right) \times H^{-1} \cap L^{1}\left(\boldsymbol{R}^{N}\right)$ initial data but, as it will become clear during the proofs, a similar analysis allows to obtain the asymptotic expansion of $\left(u, u_{t}\right)$ in $H^{s}\left(\mathbb{R}^{N}\right) \times H^{s-1}\left(\mathbb{R}^{N}\right)$ with initial data in $H^{s} \cap L^{1}\left(\mathbb{R}^{N}\right) \times H^{s-1} \cap L^{1}\left(\boldsymbol{R}^{N}\right)$. An asymptotic expansion in $L^{\infty}\left(\mathbb{R}^{N}\right)$ can also be given, but here, in the absence of the parabolic regularizing effect used in ${ }^{7}$, we should consider sufficiently smooth initial data so that the asymptotic expansion holds in $H^{s}$ with $s>0$ large enough so that $H^{s} \hookrightarrow C^{0}$.

\subsection{Main results}

The well-posedness of the equation (1.1) under the conditions (1.2) and (1.3) can be easily obtained writing (1.1) as an abstract evolution equation in the space of finite energy $H=H^{1}\left(\boldsymbol{R}^{N}\right) \times L^{2}\left(\boldsymbol{R}^{N}\right)$, with the inner product

$$
((u, v),(\widetilde{u}, \widetilde{v}))_{H}=\int_{R^{N}} u \widetilde{u} d x+\int_{R^{N}} a_{k \ell}(x) \frac{\partial u}{\partial x_{k}} \frac{\partial \widetilde{u}}{\partial x_{\ell}} d x+\int_{R^{N}} v \widetilde{v} \rho(x) d x,
$$

with $\left\{a_{k \ell}\right\}$ as in (1.2) and $\rho$ as in (1.3), whenever $(u, v),(\widetilde{u}, \widetilde{v}) \in H$. Under these conditions the operator associated to (1.1) is maximal and dissipative on $H$. Then, Lummer-Phillip's theorem guarantees that the operator associated to (1.1) is the infinitesimal generator of a continuous semigroup. Thus, we deduce that for any initial data $\left(\varphi^{0}, \varphi^{1}\right) \in L^{2}\left(\mathbb{R}^{N}\right) \times H^{-1}\left(\mathbb{R}^{N}\right)$ the equation (1.1) has a unique weak solution $u=u(x, t)$ such that

$$
u \in C^{0}\left(\mathbb{R}^{+}, L^{2}\left(\mathbb{R}^{N}\right)\right) \cap C^{1}\left(\mathbb{R}^{+}, H^{-1}\left(\mathbb{R}^{N}\right)\right) .
$$

Let us now state the main result. 
Theorem 1 Assume that $\varphi^{0} \in L^{1}\left(\mathbb{R}^{N}\right) \cap L^{2}\left(\mathbb{R}^{N}\right)$ and $\varphi^{1} \in L^{1}\left(\mathbb{R}^{N}\right) \cap H^{-1}\left(\mathbb{R}^{N}\right)$ with $|x|^{k+1} \varphi^{0}(x),|x|^{k+1} \varphi^{1}(x) \in L^{1}\left(\mathbb{R}^{N}\right)$ for some $k \geq 0$. Let $u=u(x, t)$ be the solution of (1.1). Then, there exist periodic functions $c_{\alpha} \in L_{\#}^{\infty}(Y)$, with $|\alpha| \leq k$, and constants $c_{\beta, n}$, with $n \leq \frac{k}{2}$ and $4 \leq|\beta| \leq 2 k$, depending on the initial data, the coefficients $\left\{a_{k \ell}\right\}$ and $\rho$, such that the solution $u$ satisfies

$$
\begin{gathered}
\left\|u(\cdot, t)-\sum_{|\alpha| \leq k} c_{\alpha}(\cdot)\left[G_{\alpha}^{*}(\cdot, t)+\sum_{n=1}^{p(\alpha)} \frac{t^{n}}{n !} \sum_{m=0}^{a(\alpha, n)} \sum_{|\beta|=4 n+2 m} c_{\beta, n}, G_{\alpha+\beta}^{*}(\cdot, t)\right]\right\| \leq c_{k} t^{-\frac{2 k+2+N}{4}} \\
\text { as } t \rightarrow \infty \text {. Here } p(\alpha)=\left[\frac{k-|\alpha|}{2}\right], a(\alpha, n)=p(\alpha)-n \\
G_{\alpha}^{*}(x, t)=\frac{1}{(2 \pi)^{N}} \int_{R^{N}} \xi^{\alpha} e^{-\frac{q_{k \ell}}{a_{0} \rho} \xi_{k} \xi_{\ell} t} e^{i x \cdot \xi} d \xi
\end{gathered}
$$

and $\bar{\rho}$ is the averaged density:

$$
\bar{\rho}=\frac{1}{(2 \pi)^{N}} \int_{Y} \rho(x) d x .
$$

We observe that $G_{\alpha}^{*}=(-i)^{|\alpha|}\left(\partial^{\alpha} G^{*} / \partial x_{\alpha}\right)$ where $G^{*}=G^{*}(x, t)$ is the fundamental solution of the underlying parabolic homogenized system

$$
\left\{\begin{array}{l}
\bar{\rho} a_{0} G_{t}^{*}-q_{k \ell} \frac{\partial^{2} G^{*}}{\partial x_{\ell} x_{k}}=0 \quad \text { in } \mathbb{R}^{N} \times(0, \infty) \\
G^{*}(x, 0)=\delta_{0}(x)
\end{array}\right.
$$

The convergence result in (1.6) indicates that, roughly, the solution $u$ of the equation (1.1) may be approximated at any order by a linear combination of the derivatives of the fundamental solution of the heat equation, modulated by the periodic functions $c_{\alpha}(\cdot)$. The role of these coefficients is to adapt the gaussian asymptotic profiles to the periodicity of the medium where the solution $u$ evolves.

We consider $v(x, t)=c_{0}(x) G^{*}(x, t)$, the first term of the asymptotic expansion of the solution of (1.1). As we shall see below (see Section 5 ), $c_{0}(\cdot)$ turns out be a constant and more precisely

$$
c_{0}=\frac{1}{a_{0} \bar{\rho}} m_{\rho}\left(u_{t}+a_{0} u\right) .
$$

It is important to observe that the mass of $v(x, t)$ with respect to the weight $\rho(\cdot)$, as $t \rightarrow \infty$, is the same as the mass of $u_{t}+a_{0} u$ associated to (1.1) which is constant in time as seen in (1.4). In fact, we have

$$
\begin{aligned}
m_{\rho}\left(v_{t}\right) & =-\frac{c_{0}}{(2 \pi)^{N}} \int_{R^{N}} \int_{R^{N}} \frac{q_{k \ell}}{a_{0} \bar{\rho}} \xi_{k} \xi_{\ell} e^{-\frac{q_{k \ell}}{a_{0} \bar{\rho}} \xi_{k} \xi_{\ell} t} e^{i x \cdot \xi} d \xi \rho(x) d x\left(x=y \sqrt{t}, \xi=\frac{\eta}{\sqrt{t}}\right) \\
& =-\frac{c_{0}}{t} \int_{R^{N}} \rho(y \sqrt{t}) \frac{1}{(2 \pi)^{N}} \int_{R^{N}} \frac{q_{k \ell}}{a_{0} \bar{\rho}} \eta_{k} \eta_{\ell} e^{-\frac{q_{k \ell}}{a_{0} \bar{\rho}} \eta_{k} \eta_{\ell}} e^{i y \cdot \eta} d \eta d y
\end{aligned}
$$


and

$$
m_{\rho}(v)=c_{0} \int_{R^{N}} \rho(y \sqrt{t}) \frac{1}{(2 \pi)^{N}} \int_{R^{N}} e^{-\frac{q_{k \ell}}{a_{0} \bar{\rho}} \eta_{k} \eta_{\ell}} e^{i y \cdot \eta} d \eta d y .
$$

Since $\rho(y \sqrt{t}) \rightarrow \bar{\rho}$ weakly-* in $L^{\infty}\left(\mathbb{R}^{N}\right)$ as $t \rightarrow \infty$, then

$$
m_{\rho}\left(v_{t}\right) \rightarrow 0 \quad \text { as } t \rightarrow \infty,
$$

and, thanks to

$$
\frac{1}{(2 \pi)^{N}} \int_{R^{N}} \int_{R^{N}} e^{-\frac{q_{k \ell}}{a_{0} \bar{\rho}} \eta_{k} \eta_{\ell}} e^{i y \cdot \eta} d \eta d y=1
$$

we have

$$
m_{\rho}(v) \rightarrow \bar{\rho} c_{0}, \quad \text { as } t \rightarrow \infty .
$$

Thus, we obtain as $t \rightarrow \infty$,

$$
m_{\rho}\left(v_{t}+a_{0} v\right) \longrightarrow a_{o} \bar{\rho} c_{0}=m_{\rho}\left(u_{t}+a_{0} u\right) .
$$

Therefore, as expected, the total mass of the solution is captured by the first term in the asymptotic expansion.

The rest of the paper is organized as follows. First, in Section 2 and 3, we study the simpler problem in which $\rho \equiv 1$ :

$$
\left\{\begin{array}{l}
u_{t t}-\frac{\partial}{\partial x_{k}}\left(a_{k \ell}(x) \frac{\partial u}{\partial x_{\ell}}\right)+a_{0} u_{t}=0 \quad \text { in } \boldsymbol{R}^{N} \times(0, \infty) \\
u(x, 0)=\varphi^{0}(x) \\
u_{t}(x, 0)=\varphi^{1}(x) .
\end{array}\right.
$$

In Section 2 we recall some basic results on Bloch wave decomposition. In Section 3 we prove some basic lemmas and obtain the asymptotic behavior of the solutions of (1.11). In Section 4 we prove the main result, Theorem 1, in the general case. Finally, in Section 5, we analyze the periodic functions $c_{\alpha}$ and the constants $c_{\beta, n}$ entering in the asymptotic expansion.

\section{Bloch wave decomposition}

All along this section we assume that $\rho \equiv 1$. The general case will be discussed in Section 4.

In this section we recall some basic results on Bloch wave decompositions. We refer to ${ }^{3}$ and to ${ }^{4}$ for the notations and the proofs.

Let us condider the following spectral problem parametrized by $\xi \in \mathbb{R}^{N}$ : To find $\lambda=\lambda(\xi) \in \boldsymbol{R}$ and $\psi=\psi(x ; \xi)$ (no identically zero) such that

$$
\left\{\begin{array}{l}
A \psi(\cdot ; \xi)=\lambda(\xi) \psi(\cdot ; \xi) \text { in } \mathbb{R}^{N}, \\
\psi(\cdot ; \xi) \text { is }(\xi, Y) \text {-periodic, i.e. }, \\
\psi(y+2 \pi m ; \xi)=e^{2 \pi i m \cdot \xi} \psi(y) \quad \forall m \in Z^{N}, y \in \mathbb{R}^{N},
\end{array}\right.
$$


where $A$ is the elliptic operator in divergence form

$$
A \stackrel{\text { def }}{=}-\frac{\partial}{\partial x_{k}}\left(a_{k \ell}(x) \frac{\partial}{\partial x_{\ell}}\right), \quad y \in \mathbb{R}^{N} .
$$

We can write $\psi(x ; \xi)=e^{i x \cdot \xi} \phi(x, \xi), \phi$ being $Y$-periodic in the variable $x$. It is clear from $(2.12)$ that the $(\xi, Y)$-periodicity is unaltered if we replace $\xi$ by $(\xi+m)$ with $m \in Z^{N}$. Therefore, $\xi$ can be confined to the dual cell $\xi \in Y^{\prime}=\left[-\frac{1}{2}, \frac{1}{2}\left[^{N}\right.\right.$. Under these conditions, it is known (see ${ }^{4}$ ) that the above spectral problem admits a discrete sequence of eigenvalues with the following properties:

$$
\left\{\begin{array}{l}
0 \leq \lambda_{1}(\xi) \leq \cdots \leq \lambda_{m}(\xi) \leq \cdots \rightarrow \infty \\
\lambda_{m}(\xi) \text { is a Lipschitz function of } \xi \in Y^{\prime}, \forall m \geq 1 .
\end{array}\right.
$$

Besides, the corresponding eigenfunctions denoted by $\psi_{m}(\cdot ; \xi)$ and $\phi_{m}(\cdot ; \xi)$, form orthonormal basis in the subspaces of $L_{l o c}^{2}\left(\mathbb{R}^{N}\right)$ which are $(\xi, Y)$-periodic and $Y$ periodic, respectively. Moreover, as a consequence of the min-max principle, it follows that $\left(\right.$ see $\left.^{4}\right)$

$$
\lambda_{2}(\xi) \geq \lambda_{2}^{(N)}>0, \quad \forall \xi \in Y^{\prime},
$$

where $\lambda_{2}^{(N)}$ is the second eigenvalue of $A$ in the cell $Y$ with Neumann boundary conditions on $\partial Y$.

The Bloch waves introduced above enable us to describe the spectral resolution of the unbounded self-adjoint operator $A$ in $L^{2}\left(\boldsymbol{R}^{N}\right)$, in the orthogonal basis of Bloch waves

$$
\left\{\psi_{m}(x ; \xi)=e^{i x \cdot \xi} \phi_{m}(x ; \xi): m \geq 1, \xi \in Y^{\prime}\right\}
$$

Thus, we have

Proposition 1 Let $g \in L^{2}\left(\mathbb{R}^{N}\right)$. The $m^{\text {th }}$ Bloch coefficient of $g$ is defined as follows:

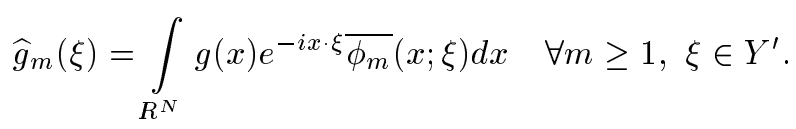

Then the following inverse formula holds:

$$
g(x)=\int_{Y^{\prime}} \sum_{m=1}^{\infty} \widehat{g}_{m}(\xi) e^{i x \cdot \xi} \phi_{m}(x ; \xi) d \xi .
$$

Further, we have Parseval's identity:

$$
\int_{R^{N}}|g(x)|^{2} d x=\int_{Y^{\prime}} \sum_{m=1}^{\infty}\left|\widehat{g}_{m}(\xi)\right|^{2} d \xi .
$$

Finally, for all $g$ in the domain of $A$, we have

$$
A g(x)=\int_{Y^{\prime}} \sum_{=1}^{\infty} \lambda_{m}(\xi) \widehat{g}_{m}(\xi) e^{i x \cdot \xi} \phi_{m}(x ; \xi) d \xi,
$$


and, consequently, the equivalence of norms in $H^{1}\left(\boldsymbol{R}^{N}\right)$ and in $H^{-1}\left(\mathbb{R}^{N}\right)$ :

$$
\begin{aligned}
\|g\|_{H^{1}\left(R^{N}\right)}^{2} & =\int_{Y^{\prime}} \sum_{m=1}^{\infty}\left(1+\lambda_{m}(\xi)\right)\left|\widehat{g}_{m}(\xi)\right|^{2} d \xi, \\
\|g\|_{H^{-1}\left(R^{N}\right)}^{2} & =\int_{Y^{\prime}} \sum_{m=1}^{\infty} \frac{\left|\widehat{g}_{m}(\xi)\right|^{2}}{1+\lambda_{m}(\xi)} d \xi .
\end{aligned}
$$

Using Proposition 1, the solution of (1.11) can be written as follows.

Lemma 1 Let $u(x, t)$ be the solution of (1.11). Then

$$
u(x, t)=\int_{Y^{\prime}} \sum_{m=1}^{\infty}\left(\beta_{m}^{1}(\xi) e^{-\alpha_{m}^{1}(\xi) t}+\beta_{m}^{2}(\xi) e^{-\alpha_{m}^{2}(\xi) t}\right) e^{i x \cdot \xi} \phi_{m}(x, \xi) d \xi,
$$

where

$$
\begin{aligned}
& \alpha_{m}^{1}(\xi)=\frac{1}{2}\left(a_{0}-\sqrt{a_{0}^{2}-4 \lambda_{m}(\xi)}\right) \\
& \alpha_{m}^{2}(\xi)=\frac{1}{2}\left(a_{0}+\sqrt{a_{0}^{2}-4 \lambda_{m}(\xi)}\right),
\end{aligned}
$$

and

$$
\begin{aligned}
& \beta_{m}^{1}(\xi)=\frac{\alpha_{m}^{2}(\xi)}{\sqrt{a_{0}^{2}-4 \lambda_{m}(\xi)}} \widehat{\varphi}_{m}^{0}(\xi)+\frac{1}{\sqrt{a_{0}^{2}-4 \lambda_{m}(\xi)}} \widehat{\varphi}_{m}^{1}(\xi), \\
& \beta_{m}^{2}(\xi)=-\frac{\alpha_{m}^{1}(\xi)}{\sqrt{a_{0}^{2}-4 \lambda_{m}(\xi)}} \widehat{\varphi}_{m}^{0}(\xi)-\frac{1}{\sqrt{a_{0}^{2}-4 \lambda_{m}(\xi)}} \widehat{\varphi}_{m}^{1}(\xi),
\end{aligned}
$$

where $\widehat{\varphi}_{m}^{0}(\xi)$ and $\widehat{\varphi}_{m}^{1}(\xi)$ are the Bloch coefficients of the initial data $\varphi^{0}$ and $\varphi^{1}$.

Proof. Since $u(x, t) \in L^{2}\left(\boldsymbol{R}^{N}\right)$ for all $t>0$, we have that

$$
u(x, t)=\int_{Y^{\prime}} \sum_{m=1}^{+\infty} \widehat{u}_{m}(\xi, t) e^{i x \cdot \xi} \phi_{m}(x, \xi) d \xi,
$$

where $\widehat{u}_{m}(\xi, t)$ is defined by Proposition 1 and satisfies for any $m \geq 1$ the following differential equation

$$
\left\{\begin{array}{l}
\partial_{t}^{2} \widehat{u}_{m}+a_{0} \partial_{t} \widehat{u}_{m}+\lambda_{m}(\xi) \widehat{u}_{m}=0 \text { in } Y^{\prime} \times(0,+\infty) \\
\widehat{u}_{m}(\cdot, 0)=\widehat{\varphi}_{m}^{0}, \frac{\partial \widehat{u}_{m}}{\partial t}(\cdot, 0)=\widehat{\varphi}_{m}^{1} \text { in } Y^{\prime} .
\end{array}\right.
$$

Here, $\partial_{t}$ denotes the derivative with respect to $t$. Solving the differential equation (2.22) we find

$$
\widehat{u}_{m}(\xi, t)=\beta_{m}^{1}(\xi) e^{-\alpha_{m}^{1}(\xi) t}+\beta_{m}^{2}(\xi) e^{-\alpha_{m}^{2}(\xi) t}
$$


where $\left\{\alpha_{m}^{i}(\xi), i=1,2\right\}$ are defined by (2.17) and (2.18), and they are the two roots of the characteric equation

$$
y^{2}+a_{0} y+\lambda_{m}(\xi)=0 .
$$

The constants $\left\{\beta_{m}^{i}(\xi), i=1,2\right\}$ as in (2.19)-(2.20) are obtained in order to meet the initial data in (1.11) $\square$.

We also have the following result on the dependence of $\lambda_{1}$ and $\phi_{1}$ with respect to the parameter $\xi$ (see ${ }^{4}$ and ${ }^{2}$ ).

Proposition 2 Assume that the coefficients $a_{k \ell}$ satisfy (1.2). Then there exists $\delta_{1}>0$ such that the first eigenvalue $\lambda_{1}$ is an analytic function on $B_{\delta_{1}}=\{\xi:|\xi|<$ $\left.\delta_{1}\right\}$ and satisfies

$$
c_{1}|\xi|^{2} \leq \lambda_{1}(\xi) \leq c_{2}|\xi|^{2}, \quad \forall \xi \in Y^{\prime}
$$

and

$$
\begin{aligned}
\lambda_{1}(0)=\partial_{k} \lambda_{1}(0)=0, & k=1, \ldots, N, \\
\partial_{k \ell}^{2} \lambda_{1}(0)=2 q_{k \ell}, & k, \ell=1, \ldots, N, \\
\partial^{\alpha} \lambda_{1}(0)=0 & \forall \alpha \text { such that }|\alpha| \text { is odd } .
\end{aligned}
$$

Futhermore, there is a choice of the first eigenfunction $\phi_{1}(x, \xi)$ satisfying

$$
\left\{\begin{array}{l}
\xi \rightarrow \phi_{1}(x, \xi) \in L^{\infty} \cap H_{\#}^{1}(Y) \text { is analytic on } B_{\delta_{1}} \\
\phi_{1}(x, 0)=(2 \pi)^{-\frac{N}{2}}
\end{array}\right.
$$

The coefficients $q_{k \ell}$ are those of the homogenized matrix associated with the family $\left(a_{k \ell}^{\varepsilon}\right)$, where $a_{k \ell}^{\varepsilon}(x)=a_{k \ell}(x / \varepsilon)$ as $\varepsilon \rightarrow 0$. Since $\alpha_{1}^{1}(\xi)$ and $\beta_{1}^{1}(\xi)$ is defined by (2.19), respectively, we have:

Proposition 3 Assume the same hypotheses as in Proposition 2. Then there $e^{-}$ xists $\delta>0$, with $\delta \leq \delta_{1}$, such that $\alpha_{1}^{1}(\xi)$ and $\beta_{1}^{1}(\xi)$ are analytic functions on $B_{\delta}$. Futhermore, $\alpha_{1}^{1}(\xi)$ satisfies

$$
c_{3}|\xi|^{2} \leq \alpha_{1}^{1}(\xi) \leq c_{4}|\xi|^{2}, \quad \forall \xi \in B_{\delta}
$$

and

$$
\begin{array}{rl}
\alpha_{1}^{1}(0)=\partial_{k} \alpha_{1}^{1}(0)=0 & k=1, \cdots, N, \\
\partial_{k \ell}^{2} \alpha_{1}^{1}(0)=2 \frac{q_{k \ell}}{a_{0}}, & k, \ell=1, \cdots, N, \\
\partial^{\beta} \alpha_{1}^{1}(0)=0 & \forall \beta \text { such that }|\beta| \text { is odd } .
\end{array}
$$




\section{Asymptotic expansion when $\rho \equiv 1$}

\subsection{Bloch component of $u$ with exponential decay}

We start this section proving that, in (2.16), the terms corresponding to the eigenvalues $\lambda_{m}(\xi), m \geq 2$, decay exponentially as $t \rightarrow \infty$. Further, we also prove that the term corresponding to $\lambda_{1}(\xi)$ goes to zero exponentially, a $t \rightarrow \infty$, whenever $\xi \in U=\left\{\xi \in Y^{\prime}:|\xi|>\delta\right\}$, with $\delta>0$.

Lemma 2 Let $\widehat{u}_{m}=\widehat{u}_{m}(\xi, t), m \geq 1$, be the Bloch coefficients associated to the solution $u=u(x, t)$ of (1.11) given in (2.16). Then, there exists positive constants $\alpha$ and $\beta$, such that

$$
\int_{Y^{\prime}} \sum_{m=2}^{\infty}\left|\widehat{u}_{m}(\xi, t)\right|^{2} d \xi \leq \alpha e^{-\beta t}\left(\left\|\varphi^{0}\right\|^{2}+\left\|\varphi^{1}\right\|_{H^{-1}}^{2}\right) .
$$

Proof. We consider the Lyapunov function

$$
L_{m}(\xi, t)=E_{m}(\xi, t)+\varepsilon F_{m}(\xi, t),
$$

where

$$
\begin{aligned}
E_{m}(\xi, t) & =\frac{1}{2}\left(\left|\partial_{t} \widehat{u}_{m}(\xi, t)\right|^{2}+\lambda_{m}(\xi)\left|\widehat{u}_{m}(\xi, t)\right|^{2}\right), \\
F_{m}(\xi, t) & =\partial_{t} \widehat{u}_{m}(\xi, t) \overline{\widehat{u}_{m}}(\xi, t)+\frac{a_{0}}{2}\left|\widehat{u}_{m}(\xi, t)\right|^{2},
\end{aligned}
$$

and $\varepsilon$ is a suitable constant to be chosen later. Here, $:$ denotes the complex conjugate. It follows from (2.15) that for $m \geq 2$,

$$
\begin{aligned}
\left|L_{m}(\xi, t)-E_{m}(\xi, t)\right| & \leq \varepsilon\left[\frac{1}{2 \lambda_{2}^{(N)}}\left|\partial_{t} \widehat{u}_{m}(\xi, t)\right|^{2}+\left(\frac{\lambda_{m}(\xi)}{2}+\frac{a_{0} \lambda_{m}(\xi)}{2 \lambda_{2}^{(N)}}\right)\left|\widehat{u}_{m}(\xi, t)\right|^{2}\right] \\
& \leq \varepsilon c_{0} E_{m}(\xi, t), \quad \text { with } c_{0}=\max \left(\frac{1}{\lambda_{2}^{(N)}}, \frac{a_{0}}{\lambda_{2}^{(N)}}+1\right)
\end{aligned}
$$

Consequently, we have for $\varepsilon<1 / c_{0}$ that

$$
\left(1-\varepsilon c_{0}\right) E_{m}(\xi, t) \leq L_{m}(\xi, t) \leq\left(1+\varepsilon c_{0}\right) E_{m}(\xi, t) .
$$

Now, we claim that

$$
\partial_{t} L_{m}(\xi, t) \leq-c L_{m}(\xi, t)
$$

holds for some positive constant independent of $\xi$ whenever $m \geq 2$. Clearly, from (3.29) and (3.30) we conclude the proof of (3.28) for all $m \geq 2$.

In order to prove the claim, we proceed as follows: Multiplying the equation (2.22) by $\overline{\partial_{t} \widehat{u}_{m}}(\xi, t)$, we obtain

$$
\partial_{t} E_{m}(\xi, t)=-a_{0}\left|\partial_{t} \widehat{u}_{m}(\xi, t)\right|^{2} .
$$


Next, we multiply the equation $(2.22)$ by $\overline{\widehat{u}_{m}}(\xi, t)$ to obtain

$$
\partial_{t}\left(\partial_{t} \widehat{u}_{m}(\xi, t) \overline{\widehat{u}_{m}}(\xi, t)+\frac{a_{0}}{2}\left|\widehat{u}_{m}(\xi, t)\right|^{2}\right)=-\lambda_{m}(\xi)\left|\widehat{u}_{m}(\xi, t)\right|^{2}+\left|\partial_{t} \widehat{u}_{m}(\xi, t)\right|^{2}
$$

Adding the identities above and choosing $\varepsilon$ small we deduce that

$$
\begin{aligned}
\partial_{t} L_{m}(\xi, t) & =-a_{0}\left|\partial_{t} \widehat{u}_{m}(\xi, t)\right|^{2}-\varepsilon \lambda_{m}(\xi)\left|\widehat{u}_{m}(\xi, t)\right|^{2}+\varepsilon\left|\partial_{t} \widehat{u}_{m}(\xi, t)\right|^{2} \\
& \leq-c_{1}(\varepsilon) E_{m}(\xi, t) \quad \text { with } 0<c_{1}(\varepsilon)=2 \min \left(\varepsilon, a_{0}-\varepsilon\right)
\end{aligned}
$$

Now, using (3.29) with $\varepsilon$ sufficiently small and satisfying (3.31), in particular $\varepsilon<a_{0}$, there exits $c_{2}=c_{2}(\varepsilon)>0$ such that

$$
\partial_{t} L_{m}(\xi, t) \leq-c_{2} L_{m}(\xi, t), \text { where } c_{2}=\frac{c_{1}}{1-\varepsilon\left(c_{0}\right)^{-1}}>0 .
$$

Therefore,

$$
L_{m}(\xi, t) \leq L_{m}(\xi, 0) e^{-c_{2} t},
$$

and using again (3.29) we obtain

$$
E_{m}(\xi, t) \leq c E_{m}(\xi, 0) e^{-c_{2} t},
$$

where $c=1+\varepsilon\left(c_{0}\right)^{-1}$. Recalling the definition of $E_{m}(\xi, t)$ we have from (3.32) and (2.14) that

$$
\lambda_{m}(\xi)\left|\widehat{u}_{m}(\xi, t)\right|^{2} \leq c e^{-c_{2} t}\left\{\left|\partial_{t} \widehat{u}_{m}(\xi, 0)\right|^{2}+\lambda_{m}(\xi)\left|\widehat{u}_{m}(\xi, 0)\right|^{2}\right\}
$$

and

$$
\left|\widehat{u}_{m}(\xi, t)\right|^{2} \leq c e^{-c_{2} t}\left\{\frac{1}{\lambda_{2}^{(N)}}\left|\partial_{t} \widehat{u}_{m}(\xi, 0)\right|^{2}+\left|\widehat{u}_{m}(\xi, 0)\right|^{2}\right\} .
$$

Adding the inequalities above it follows that

$$
\left(1+\lambda_{m}(\xi)\right)\left|\widehat{u}_{m}(\xi, t)\right|^{2} \leq c e^{-c_{2} t}\left(1+\frac{1}{\lambda_{2}^{(N)}}\right)\left\{\left|\partial_{t} \widehat{u}_{m}(\xi, 0)\right|^{2}+\left(1+\lambda_{m}(\xi)\right)\left|\widehat{u}_{m}(\xi, 0)\right|^{2}\right\}
$$

and consequently

$$
\left|\widehat{u}_{m}(\xi, t)\right|^{2} \leq c e^{-c_{2} t}\left(1+\frac{1}{\lambda_{2}^{(N)}}\right)\left\{\left|\widehat{u}_{m}(\xi, 0)\right|^{2}+\frac{\left|\partial_{t} \widehat{u}_{m}(\xi, 0)\right|^{2}}{1+\lambda_{m}(\xi)}\right\} .
$$

Therefore,

$$
\int_{Y^{\prime}} \sum_{m=2}^{\infty}\left|\widehat{u}_{m}(\xi, t)\right|^{2} d \xi \leq c_{3} e^{-c_{2} t} \int_{Y^{\prime}} \sum_{m=2}^{\infty}\left\{\left|\widehat{\varphi}_{m}^{0}(\xi)\right|^{2}+\frac{\left|\widehat{\varphi}_{m}^{1}(\xi)\right|^{2}}{1+\lambda_{m}(\xi)}\right\} d \xi,
$$

where $\widehat{\varphi}_{m}^{0}(\xi)$ and $\widehat{\varphi}_{m}^{1}(\xi)$ are the Bloch coefficients of the initial data $\varphi^{0}$ and $\varphi^{1}$, respectively. This completes the proof of (3.28) $\square$. 
Remark 1 We choose $\delta>0$ in Proposition 3 such that $\delta \leq \delta_{1}$ ( $\delta_{1}$ being the radius of the ball where $\lambda_{1}$ and $\phi_{1}$ are analytic as in Proposition 2) and satisfying that

$$
a_{0}^{2}-4 \lambda_{1}(\xi) \geq a_{0}^{2}-4 c_{2} \delta^{2}=c_{\delta}>0, \forall \xi \in B_{\delta}
$$

Obviously this can be done since $\lambda_{1}(0)=\nabla_{\xi} \lambda(0)=0$.

Lemma 3 Let $\widehat{u}_{1}=\widehat{u}_{1}(\xi, t)$ be the first Bloch coefficient of the solution $u$ of (1.11) given in (2.16). Then, there exist positive constants $\alpha$ and $\beta$, such that

$$
\int_{Y^{\prime}-B_{\delta}}\left|\widehat{u}_{1}(\xi, t)\right|^{2} d \xi \leq \alpha e^{-\beta t}\left(\left\|\varphi^{0}\right\|^{2}+\left\|\varphi^{1}\right\|_{H^{-1}}^{2}\right),
$$

with $\delta$ satisfying (3.33). On the other hand, the second component $\beta_{1}^{2}(\xi) e^{-\alpha_{1}^{2}(\xi) t}$ of $\widehat{u}_{1}(\xi, t)$ as in (2.23) satisfies

$$
\int_{B_{\delta}}\left|\beta_{1}^{2}(\xi)\right|^{2} e^{-2 \alpha_{1}^{2}(\xi) t} d \xi \leq C e^{-a_{0} t}\left(\left\|\varphi^{0}\right\|^{2}+\left\|\varphi^{1}\right\|_{H^{-1}}^{2}\right) .
$$

Proof. In order to prove (3.34) we argue as in Lemma 2. We consider the Lyapunov fuction $L_{1}(\xi, t)$ and, instead of $(2.15)$, we use Proposition 2 and Remark 1 to obtain (3.29) for $\xi \in Y^{\prime}-B_{\delta}$ and $m=1$. We observe that (2.24) gives us

$$
\frac{1}{\lambda_{1}(\xi)}<\frac{1}{c_{1} \delta^{2}}, \quad \forall \xi \in Y^{\prime}-B_{\delta},
$$

and (3.34) is obtained following the same steps of Lemma 2.

To prove (3.35) it is enough to observe that, thanks to (3.33), and since, according to $(2.18) \alpha_{1}^{2}(\xi) \geq a_{0} / 2$ for all $\xi \in B_{\delta}$, we have

$$
\begin{aligned}
&\left(1+\lambda_{1}(\xi)\right)\left|\beta_{1}^{2}(\xi)\right|^{2} e^{-2 \alpha_{1}^{2}(\xi) t} \leq\left(1+c_{2}|\xi|^{2}\right)\left|\beta_{1}^{2}(\xi)\right|^{2} e^{-2 \alpha_{1}^{1}(\xi) t} \\
& \quad \leq\left(1+c_{2} \delta^{2}\right) \frac{4+\left(a_{0}-\sqrt{c_{\delta}}\right)^{2}}{2 c_{\delta}} e^{-a_{0} t}\left(\left|\widehat{\varphi}_{0}^{1}(\xi)\right|^{2}+\left|\widehat{\varphi}_{1}^{1}(\xi)\right|^{2}\right) \\
& \leq\left(1+c_{2} \delta^{2}\right) \frac{4+\left(a_{0}-\sqrt{c_{\delta}}\right)^{2}}{2 c_{\delta}} e^{-a_{0} t}\left(\left(1+\lambda_{1}(\xi)\right)\left|\widehat{\varphi}_{0}^{1}\right|^{2}+\left|\widehat{\varphi}_{1}^{1}\right|^{2}\right) .
\end{aligned}
$$

Consequently,

$$
\left|\beta_{1}^{2}(\xi)\right|^{2} e^{-2 \alpha_{1}^{2}(\xi) t} \leq C(\delta) e^{-a_{0} t}\left(\left|\widehat{\varphi}_{1}^{0}(\xi)\right|^{2}+\frac{\left|\widehat{\varphi}_{1}^{1}(\xi)\right|^{2}}{1+\lambda_{1}(\xi)}\right),
$$

and the result follows $\square$.

\subsection{Bloch component of $u$ with polynomial decay}


Thanks to Lemmas 2 and 3, to conclude the proof of the Theorem 1 it is sufficient to analyse

$$
I(x, t)=\int_{B_{\delta}} \beta_{1}^{1}(\xi) e^{-\alpha_{1}^{1}(\xi) t} e^{i x \cdot \xi} \phi_{1}(x ; \xi) d \xi,
$$

since the other components of $u$ have an exponentially decay in $L^{2}\left(\mathbb{R}^{N}\right)$. To do that we make use of classical asymptotic lemmas (see Lemma 5 and Lemma 6 below) and assume that the initial data $\varphi^{0} \in L^{1}\left(\mathbb{R}^{N}\right)$ and $\varphi^{1} \in L^{1}\left(\mathbb{R}^{N}\right) \cap H^{-1}\left(\mathbb{R}^{N}\right)$ are such that $|x|^{k+1} \varphi^{0}(x),|x|^{k+1} \varphi^{1}(x) \in L^{1}\left(\boldsymbol{R}^{N}\right)$. Under these conditions the first Bloch coefficients $\widehat{\varphi}_{1}^{0}(\xi)$ and $\widehat{\varphi}_{1}^{1}(\xi)$ of the initial data belong to $C^{k+1}\left(B_{\delta}\right)$ (see Lemma 4 ), what is crucial in the proof of the asymptotic expansion (see the definitions of $\beta_{1}^{1}(\xi)$ and $\alpha_{1}^{1}(\xi)$ in Lemma 1$)$.

Lemma 4 Let $\varphi \in L^{1}\left(\mathbb{R}^{N}\right)$ be a function such that $|x|^{k} \varphi \in L^{1}\left(\boldsymbol{R}^{N}\right)$. Then, its first Bloch coefficient $\widehat{\varphi}_{1}(\xi)$, belongs to $C^{k}\left(B_{\delta}\right)$, where $B_{\delta}$ is a neighborhood of $\xi=0$ where the first Bloch wave $\phi_{1}(x ; \xi)$ is analytic.

Proof. Since

$$
\widehat{\varphi}_{1}(\xi)=\int_{R^{N}} \varphi(x) e^{-i \cdot \xi} \overline{\phi_{1}}(x ; \xi) d x,
$$

for all $\alpha \in(N \cup\{0\})^{N}$ with $|\alpha| \leq k$, we have

$$
\frac{\partial \widehat{\varphi}_{1}}{\partial \xi_{\alpha}}(\xi)=\sum_{\beta \leq \alpha}\left(\begin{array}{c}
\alpha \\
\beta
\end{array}\right) \int_{R^{N}} \varphi(x)(-i)^{|\beta|} x^{\beta} e^{-i \cdot \xi} \frac{\partial \overline{\phi_{1}}}{\partial \xi_{\alpha-\beta}}(x ; \xi) d x,
$$

where $\beta \leq \alpha$ if and only if $\beta_{k} \leq \alpha_{k}$ for all $j=1, \ldots, N$, and

$$
\left(\begin{array}{c}
\alpha \\
\beta
\end{array}\right)=\prod_{k=1}^{N}\left(\begin{array}{c}
\alpha_{j} \\
\beta_{j}
\end{array}\right) \text {. }
$$

Moreover, Proposition 2 gives us that the function $\xi \rightarrow \phi_{1}(x ; \xi)$ is analytic with values in $L_{\#}^{\infty}(Y)$, what guarantees that

$$
\begin{aligned}
\left|\frac{\partial \widehat{\varphi}_{1}}{\partial \xi_{\alpha}}(\xi)\right| & \leq \sum_{\beta \leq \alpha}\left(\begin{array}{c}
\alpha \\
\beta
\end{array}\right) c_{\beta} \int_{R^{N}}\left|\varphi(x) x^{\beta}\right| d x \\
& \leq \sum_{\beta \leq \alpha}\left(\begin{array}{c}
\alpha \\
\beta
\end{array}\right) c_{\beta} \int_{R^{N}}\left(1+|x|^{k}\right)|\varphi(x)| d x
\end{aligned}
$$

for $0 \leq|\alpha| \leq k$, where $c_{\beta}$ is a positive constant. Thus, using again Proposition 2, we have that the map $\xi \rightarrow e^{-i \xi \cdot x} \partial_{\xi}^{\alpha} \phi_{1}(x ; \xi)$ is continuous, and the result follows $\square$.

Now, we are going to present some basic asymptotic results. The following definition will be useful to simplify the notation. 
Definition 1 Given $f, g \in C(\mathbb{R} ; \mathbb{R})$, we say that $f$ and $g$ are of the same order as $t \rightarrow \infty$ and we denote it by $f \sim g$ when

$$
\lim _{t \rightarrow \infty} \frac{f(t)}{g(t)}=1
$$

The following basic lemmas on asymptotic analysis are needed (see ${ }^{1}$, p. 263 ):

Lemma 5 Let $f:[0, b] \rightarrow \boldsymbol{R}$ be a continuous function such that it has the uniform asymtotic series expansion

$$
f(x)=x^{\alpha} \sum_{n=1}^{\infty} a_{n} x^{\beta_{n}}, x \in[0, b],
$$

with $\alpha>1$ and $\beta_{n}>0$. Then

$$
\int_{0}^{b} e^{-t x} f(x) d x \sim \sum_{n=1}^{\infty} a_{n} \frac{\Gamma\left(\alpha+\beta_{n}+1\right)}{t^{\left(\alpha+\beta_{n}+1\right)}}, \text { as } t \rightarrow \infty .
$$

When $f(x)=x^{\alpha}$ we have that

$$
\int_{0}^{b} e^{-t x} x^{\alpha} d x \sim \frac{\Gamma(\alpha+1)}{t^{(\alpha+1)}}, \text { as } t \rightarrow \infty
$$

where

$$
\Gamma(z)=\int_{0}^{\infty} e^{-x} x^{z-1} d x, z>0 .
$$

As a consequence of Lemma 5 , the following holds in ${ }^{7}$ :

Lemma 6 Let $c>0$. Then

$$
\int_{b_{\delta}} e^{-c|\xi|^{2} t}|\xi|^{k} d \xi \sim c_{k} t^{-\frac{k+N}{2}}, \text { as } t \rightarrow \infty,
$$

for all $k \in N$, where $c_{k}$ is a positive constant that may be computed explicitly. On the other hand, if $q=\left(q_{i j}\right)$ is a symmetric positive matrix, we also have

$$
\int_{Y^{\prime}} e^{-\frac{q_{i j} j}{a_{0}} \xi_{i} \xi_{j} t} \xi^{\beta} d \xi \sim c_{|\beta|} t^{-\frac{(|\beta|+N)}{2}}, \text { as } t \rightarrow \infty,
$$

for all multi-index $\beta \in(N \cup\{0\})^{N}$ and for a suitable constant $c_{|\beta|}$ that may computed as well.

In the sequel we prove three lemmas which are related to the asymptotic behavior of the terms $\beta_{1}^{1}(\xi), \phi_{1}(x ; \xi)$ and $e^{-\alpha_{1}^{1}(\xi) t}$, respectively, that appear in (3.36). 
We recall that the idea we have in mind is to prove that the solutions of (1.1) may be approximated by a linear combination of the derivatives of the fundamental solution of the homogenized heat equation and, as we said in the begining of this section, in view of Lemma 5 and 6, our analysis may be restricted to consider (3.36). Thus, our first step in this direction is to prove that (3.36) can be replaced by

$$
J(x, t)=\int_{B_{\delta}} \sum_{|\alpha| \leq k} d_{\alpha} \xi^{\alpha} e^{-\alpha_{1}^{1}(\xi) t} e^{i x \cdot \xi} \phi_{1}(x ; \xi) d \xi, \quad(x, t) \in \mathbb{R}^{N} \times \mathbb{R}^{+},
$$

where

$$
d_{\alpha}=\frac{1}{\alpha !} \partial^{\alpha} \beta_{1}^{1}(0)
$$

which are obtained by means of the Taylor expansion of $\beta_{1}^{1}(\xi)$ in $\xi=0$.

Lemma 7 Let $\varphi^{0} \in L^{1}\left(\mathbb{R}^{N}\right) \cap L^{2}\left(\mathbb{R}^{N}\right)$ and $\varphi^{1} \in L^{1}\left(\mathbb{R}^{N}\right) \cap H^{-1}\left(\boldsymbol{R}^{N}\right)$ be such that $|x|^{k+1} \varphi^{0}(x),|x|^{k+1} \varphi^{1}(x) \in L^{1}\left(\mathbb{R}^{N}\right)$. Consider $I(x, t)$ as in (3.36) and $J(x, t)$ as in (3.37). Then, there exist $c_{k}>0$ such that

$$
\|I(\cdot, t)-J(\cdot, t)\| \leq c_{k} t^{-\frac{2 k+2+N}{4}} \text { as } t \rightarrow \infty .
$$

Proof. Since $\varphi^{0} \in L^{1}\left(\mathbb{R}^{N}\right) \cap L^{2}\left(\mathbb{R}^{N}\right)$ and $\varphi^{1} \in L^{1}\left(\mathbb{R}^{N}\right) \cap H^{-1}\left(\boldsymbol{R}^{N}\right)$ with $|x|^{k+1} \varphi^{0}$, $|x|^{k+1} \varphi^{1} \in L^{1}\left(\mathbb{R}^{N}\right)$ then, from Lemma 4 , we have that $\widehat{\varphi}_{1}^{0}, \widehat{\varphi}_{1}^{1} \in C^{k+1}\left(B_{\delta}\right)$, and, according to (2.19), $\beta_{1}^{1} \in C^{k+1}\left(B_{\delta}\right)$ as well. Thus, thanks to (3.38), from the Taylor expansion we have that for all $\xi \in B_{\delta}$,

$$
\left|\beta_{1}^{1}(\xi)-\sum_{|\alpha| \leq k} d_{\alpha} \xi^{\alpha}\right| \leq C_{k}|\xi|^{k+1}, \quad C_{k}>0
$$

These constants can be computed explicitly in terms of $\partial^{\alpha} \lambda_{1}(0)$ and $\partial^{\alpha} \phi_{1}(0)$, and the mass of the initial datum (see Section 5). Indeed, from Parseval's identity, we have

$$
\|I(\cdot, t)-J(\cdot, t)\|^{2}=\int_{B_{\delta}}\left|\beta_{1}^{1}(\xi)-\sum_{|\alpha| \leq k} d_{\alpha} \xi^{\alpha}\right|^{2} e^{-2 \alpha_{1}^{1}(\xi) t} d \xi .
$$

Then, thanks to estimate (2.26) in Proposition 3 and Lemma 6, we obtain

$$
\begin{aligned}
\|I(\cdot, t)-J(\cdot, t)\|^{2} & =\int_{B_{\delta}}\left|\beta_{1}^{1}(\xi)-\sum_{|\alpha| \leq k} d_{\alpha} \xi^{\alpha}\right|^{2} e^{-2 c_{3}|\xi|^{2} t} d \xi \\
& \leq c_{k} \int_{B_{\delta}}|\xi|^{2(k+1)} e^{-2 c_{3}|\xi|^{2} t} d \xi \sim c_{k} t^{-\frac{2 k+2+N}{2}}, \text { as } t \rightarrow \infty
\end{aligned}
$$

This concludes the proof of Lemma $7 \square$. 
In a second step, we compute the Taylor expansion of $\phi_{1}(x, \xi)$ around $\xi=0$, and prove that all the terms entering in the definition (3.37) of $J$ and that we denote by $J_{\alpha}$ with $\alpha \in(N \cup 0)^{N}$,

$$
J_{\alpha}(x, t)=\frac{1}{(2 \pi)^{N}} \int_{B_{\delta}} \xi^{\alpha} e^{-\alpha_{1}^{1}(\xi) t} e^{i x \cdot \xi} \phi_{1}(x ; \xi) d \xi, \quad(x, t) \in \boldsymbol{R}^{N} \times \mathbb{R}^{+},
$$

may be approximated in $L^{2}$-setting by a linear combination of the form

$$
\frac{1}{(2 \pi)^{N}} \sum_{|\gamma| \leq k-|\alpha|} \widetilde{d}_{\gamma}(x) \int_{B_{\delta}} \xi^{\alpha} e^{-\alpha_{1}^{1}(\xi) t} e^{i x \cdot \xi},
$$

where $\widetilde{d}_{\gamma}$ are periodic functions defined by

$$
\widetilde{d}_{\alpha}(\cdot)=\frac{1}{\alpha !} \partial_{\xi}^{\alpha} \phi_{1}(\cdot ; 0) \in L_{\#}^{\infty}\left(\mathbb{R}^{N}\right) .
$$

This way be done obtaining the same rate of decay as in Lemma 7 .

Now we present a result from ${ }^{2}$ that will be needed when stating and proving in Lemma 9 the facts metioned above.

Lemma 8 Let us introduce

$$
G(x)=\int_{Y^{\prime}} g(\xi) e^{i x \cdot \xi} \omega(x ; \xi) d \xi, \quad x \in \boldsymbol{R}^{N},
$$

where $g \in L^{2}\left(Y^{\prime}\right)$ and $\omega \in L^{\infty}\left(Y^{\prime} ; L_{\#}^{2}(Y)\right)$. Then we have

$$
\|G\|^{2}=\int_{Y^{\prime}}|g(\xi)|^{2}\|\omega(\cdot ; \xi)\|_{L^{2}(Y)}^{2} d \xi
$$

Proof. To check this result we expand $\omega(x ; \xi)$ as a function of $x$ in the orthonormal basis $\left\{\phi_{m}(x ; \xi)\right\}_{m=1}^{\infty}$ where $\xi \in Y^{\prime}$ is a parameter:

$$
\omega(x ; \xi)=\sum_{m=1}^{\infty} a_{m}(\xi) \phi_{m}(x ; \xi) .
$$

Introducing this expression in (3.42), we get

$$
G(x)=\int_{Y^{\prime}} g(\xi) \sum_{m=1}^{\infty} a_{m}(\xi) e^{i x \cdot \xi} \phi_{m}(x ; \xi) d \xi .
$$

Applying the Parseval's identity of Proposition 1, it follows that

$$
\|G\|^{2}=\int_{Y^{\prime}}|g(\xi)|^{2} \sum_{m=1}^{\infty}\left|a_{m}(\xi)\right|^{2} d \xi
$$


This completes the proof of the lemma if we use the Parseval's identity in $L^{2}(Y)$ :

$$
\|\omega(\cdot ; \xi)\|_{L^{2}(Y)}^{2}=\sum_{m=1}^{\infty}\left|a_{m}(\xi)\right|^{2} \quad \forall \xi \in Y^{\prime} \square .
$$

Lemma 9 We consider $J_{\alpha}(x, t)$ defined in (3.40), with $|\alpha| \leq k$, and

$$
I_{\alpha}(x, t)=\frac{1}{(2 \pi)^{N}} \int_{B_{\delta}} \xi^{\alpha} e^{-\alpha_{1}^{1}(\xi) t} e^{i x \cdot \xi} d \xi, \quad(x, t) \in \mathbb{R}^{N} \times \boldsymbol{R}^{+},
$$

Then there exits periodic functions $\widetilde{d}_{\gamma}=\widetilde{d}_{\gamma}(x)$ defined in (3.41), such that

$$
\left\|J_{\alpha}(\cdot, t)-\sum_{|\gamma| \leq k-|\alpha|} \tilde{d}_{\gamma}(\cdot) I_{\gamma+\alpha}(\cdot, t)\right\| \leq c_{k,|\alpha|} t^{-\frac{2 k+2+N}{4}} \text {, as } t \rightarrow \infty .
$$

Proof. We set

$$
R_{k}(x ; \xi)=\phi_{1}(x ; \xi)-\sum_{|\alpha| \leq k} \widetilde{d}_{\alpha}(x) \xi^{\alpha},
$$

where $\widetilde{d}_{\alpha}(\cdot)$ is defined in (3.41). Since $\phi_{1}$ is an analytic function with respect to $\xi$ in $B_{\delta}$ and values in $L^{2}(Y)$ we have, for all $\xi \in B_{\delta}$,

$$
\left\|R_{k}(\cdot ; \xi)\right\|_{L^{2}(Y)} \leq C_{k}|\xi|^{k+1}, C_{k}>0 .
$$

Thus, $R_{k} \in L^{\infty}\left(Y^{\prime} ; L_{\#}^{2}(Y)\right)$. Then, for $\alpha \in(N \cup\{0\})^{N}$ with $|\alpha| \leq k$, we have

$$
J_{\alpha}(x, t)-\sum_{|\gamma| \leq k-|\alpha|} \widetilde{d}_{\gamma}(x) I_{\gamma+\alpha}(x, t)=\frac{1}{(2 \pi)^{N}} \int_{B_{\delta}} \xi^{\alpha} e^{-\alpha_{1}^{1}(\xi) t} e^{i x \cdot \xi} R_{k}(x ; \xi) d \xi,
$$

and since $R_{k}(y ; \xi)$ is $Y$-periodic in the variable $y$, it follows from Lemma 8 that

$$
\begin{aligned}
\left\|J_{\alpha}(\cdot, t)-\sum_{|\gamma|=0}^{k-|\alpha|} \widetilde{d}_{\gamma}(\cdot) I_{\gamma+\alpha}(\cdot, t)\right\|^{2} & \leq \int_{B_{\delta}} \frac{|\xi|^{2|\alpha|}}{(2 \pi)^{2 N}} e^{-2 \alpha_{1}^{1}(\xi) t}\left\|R_{k-|\alpha|}(\cdot ; \xi)\right\|_{L^{2}(Y)}^{2} d \xi \\
& \leq C_{k-|\alpha|} \int_{B_{\delta}}|\xi|^{2 k+2} e^{-2 \alpha_{1}^{1}(\xi) t} d \xi
\end{aligned}
$$

and we conclude as in Lemma 7, using the Proposition 2 and Lemma $6 \square$.

The proofs of Lemmas 7 and 9, as well as that of Lemma 10 that we present below, provide a systematic way of computing the coefficients that appear in the statement of Theorem 1 . We note that the functions $c_{\alpha}(\cdot)$ are related to the derivatives of the first Bloch eigenfunction $\phi_{1}(x ; \xi)$ with respect to $\xi$ in $\xi=0$. We shall describe how to compute them explicitly in Section 5. 
Now, we are going to study the asymptotic behavior of the integral $I_{\alpha}(x, t)$ defined in Lemma 9. We observe that, according to Proposition 3,

$$
\begin{aligned}
\alpha_{1}^{1}(0)=\partial_{k} \alpha_{1}^{1}(0)=0, & k=1, \ldots, N, \\
\partial_{k \ell}^{2} \alpha_{1}^{1}(0)=\frac{2 q_{k \ell}}{a_{0}}, & \ell, k=1, \ldots, N,
\end{aligned}
$$

where the coefficients $q_{k \ell}$ are those of the homogenized matrix associated with the family $\left(a_{k \ell}^{\varepsilon}\right)$, where $a_{k \ell}^{\varepsilon}(x)=a_{k \ell}(x / \varepsilon)$, as $\varepsilon \rightarrow 0$. Then, for all $\xi \in B_{\delta}$, we have

$$
e^{-\alpha_{1}^{1}(\xi) t} \sim e^{-\frac{q_{k \ell}}{a_{0}} \xi_{k} \xi_{\ell} t}, \quad \text { as } t \rightarrow \infty .
$$

This fact provides a first idea of the behavior of $I_{\alpha}(x, t)$, defined in (3.43), as $t \rightarrow \infty$. Futhermore, in Lemma 10 it turns evident that solutions of (1.1) behave as a linear combination of functions $G_{\alpha}^{*}(x, t)$ introduced in (1.7), which are the derivatives $(-i)^{|\alpha|} \partial_{x}^{\alpha}$ of the fundamental solution $G^{*}$ of the homogenized heat equation.

Lemma 10 We consider the function $I_{\alpha}(x, t)$ defined in (3.43) with $|\alpha| \leq k$. Then, there exist constans $c_{\beta, n}$, with $4 \leq|\beta| \leq 4 p(\alpha)$, such that

$$
\left\|I_{\alpha}(\cdot, t)-I_{\alpha}^{*}(\cdot, t)-\sum_{n=1}^{p(\alpha)} \frac{t^{n}}{n !} \sum_{m=0}^{a(\alpha, n)} \sum_{|\beta|=4 n+2 m} c_{\beta, n} I_{\alpha+\beta}^{*}(\cdot, t)\right\| \leq c_{k,|\alpha|} t^{-\frac{2 k+2+N}{4}},
$$

as $t \rightarrow \infty$, with $p(\alpha)=\left[\frac{k-|\alpha|}{2}\right], a(\alpha, n)=p(\alpha)-n$, and where for $\alpha \in(N \cup 0)^{N}$

$$
I_{\alpha}^{*}(x, t)=\frac{1}{(2 \pi)^{N}} \int_{B_{\delta}} \xi^{\alpha} e^{-\frac{q_{k \ell}}{a_{0}} \xi_{k} \xi_{\ell} t} e^{i x \cdot \xi} d \xi, \quad(x, t) \in \mathbb{R}^{N} \times \mathbb{R}^{+} .
$$

Here and in the sequel [.] denotes the integer part.

Remark 2 In view of (1.7) with $\bar{\rho}=1$ and (3.44) the analogy between $I_{\alpha}^{*}$ and $G_{\alpha}^{*}$ is clear. $I_{\alpha}^{*}$ is in fact obtained by integrating the same quantity as in $G_{\alpha}^{*}$ but this time in $B_{\delta}$ instead of $\mathbb{R}^{N}$. In particular, $I_{\alpha}^{*}(x, t)$ has the same polynomial decay as $G_{\alpha}^{*}(x, t)$, because

$$
\left\|G_{\alpha}^{*}(\cdot, t)-I_{\alpha}^{*}(\cdot, t)\right\| \leq c e^{-c(\delta) t}, \quad \text { with } c(\delta)>0 .
$$

Indeed, by Parseval's identity and the coercitivity of coefficients $\left\{q_{k \ell}\right\}$ we have

$$
\begin{aligned}
\left\|G_{\alpha}^{*}(\cdot, t)-I_{\alpha}^{*}(\cdot, t)\right\|^{2} & =\frac{1}{(2 \pi)^{2 N}} \int_{R^{N}-B_{\delta}}|\xi|^{2|\alpha|} e^{-2 \frac{q_{k \ell}}{a_{0}} \xi_{k} \xi_{\ell} t} d \xi \\
& \leq \frac{1}{(2 \pi)^{2 N}} \int_{R^{N}-B_{\delta}}|\xi|^{2|\alpha|} d \xi e^{-2 \frac{c}{a_{0}}|\xi|^{2} t} d \xi \leq c e^{-c(\delta) t} .
\end{aligned}
$$


Proof of Lemma 10. We consider the map $\xi \in B_{\delta} \rightarrow \nu(\xi)$ given by

$$
\nu(\xi)=\alpha_{1}^{1}(\xi)-\frac{1}{2} \partial_{k \ell}^{2} \alpha_{1}^{1}(0) \xi_{k} \xi_{\ell}=\alpha_{1}^{1}(\xi)-\frac{q_{k \ell}}{a_{0}} \xi_{k} \xi_{\ell} .
$$

Thanks to Proposition 3, the map $\nu=\nu(\xi)$ is analytic in $B_{\delta}$. Moreover, it follows from (2.27) that for $\xi \in B_{\delta}$

$$
|\nu(\xi)|=\left|\alpha_{1}^{1}(\xi)-\frac{q_{k \ell}}{a_{0}} \xi_{k} \xi_{\ell}\right| \leq c|\xi|^{4} .
$$

The function $\left(e^{-\nu(\xi) t}-1\right)$ is also analytic, and by (3.47) we have

$$
\left|e^{-\nu(\xi) t}-\sum_{n=0}^{p} \frac{t^{n}}{n !}(-\nu(\xi))^{n}\right| \leq C_{p}(\nu(\xi) t)^{p+1} \leq c_{p}|\xi|^{4 p+4} t^{p+1} .
$$

Thus, defining for $p \geq 1$

$$
\nu_{\alpha, p}(x, t)=\frac{1}{(2 \pi)^{N}} \int_{B_{\delta}} \xi^{\alpha}\left[\sum_{n=0}^{p} \frac{t^{n}}{n !}(-\nu(\xi))^{n}\right] e^{-\frac{q_{k \ell}}{a_{0}} \xi_{k} \xi_{\ell} t} e^{i x \cdot \xi} d \xi,(x, t) \in \mathbb{R}^{N} \times \mathbb{R}^{+},
$$

and replacing (3.46) in $I_{\alpha}(x, t)$, we get

$$
I_{\alpha}(x, t)-\nu_{\alpha, p}(x, t)=\frac{1}{(2 \pi)^{N}} \int_{B_{\delta}} \xi^{\alpha} e^{-\frac{q_{k \ell}}{\alpha_{0}} \xi_{k} \xi_{\ell} t}\left[e^{-\nu(\xi) t}-\sum_{n=0}^{p} \frac{t^{n}}{n !}(-\nu(\xi))^{n}\right] e^{i x \cdot \xi} d \xi .
$$

Then, from Parseval's identity and (3.48) it follows that

$$
\begin{aligned}
\left\|I_{\alpha}(\cdot, t)-\nu_{\alpha, p}(\cdot, t)\right\|= & \int_{B_{\delta}} \frac{|\xi|^{2|\alpha|}}{(2 \pi)^{2 N}}\left|e^{-\nu(\xi) t}-\sum_{n=0}^{p} \frac{t^{n}}{n !}(-\nu(\xi))^{n}\right|^{2} e^{-2 \frac{q_{k} \ell}{a_{0}} \xi_{k} \xi_{\ell} t} d \xi \\
& \leq c t^{2 p+2} \int_{B_{\delta}}|\xi|^{2|\alpha|+8 p+8} e^{-2 \frac{q_{k} \ell}{a_{0}} \xi_{k} \xi_{\ell} t} d \xi \\
& \sim \widetilde{c} t^{2 p+2} t^{-\frac{2|\alpha|+8 p+8+N}{2}}=\widetilde{c} t^{-\frac{2|\alpha|+4 p+4+N}{2}} \quad \text { as } t \rightarrow \infty .
\end{aligned}
$$

Now we choose $p$ such that the above decay rate is of the order of $(2 k+2+N) / 2$, i.e., $p$ satisfies $(2|\alpha|+4 p+4+N \geq 2 k+N+2)$, or, equivalently,

$$
2 p \geq k-|\alpha|-1
$$

Thus, we have

$$
\left\|I_{\alpha}(\cdot, t)-\nu_{\alpha, p}(\cdot, t)\right\| \leq \widetilde{c} t^{-\frac{2 k+n+2}{4}}, \quad \text { as } t \rightarrow \infty .
$$


To conclude the proof we are going to study the asymptotic behavior of the integral $\nu_{\alpha, p}(x, t)$ defined above. But before doing it, we note that if we consider the Taylor expansion of $\nu(\xi)$ around $\xi=0$, we obtain

$$
\nu_{\alpha, p}(x, t)=\frac{1}{(2 \pi)^{N}} \int_{B_{\delta}} \xi^{\alpha}\left\{1+\sum_{n=1}^{p} \frac{t^{n}}{n !}\left(\sum_{|\beta|=0}^{\infty} \frac{1}{\beta !} \partial_{\xi}^{\beta} \nu(0) \xi^{\beta}\right)^{n}\right\} e^{-\frac{q_{k \ell} \ell}{a_{0}} \xi_{k} \xi_{\ell} t} e^{i x \cdot \xi} d \xi .
$$

Indeed, since $\partial^{\beta} \nu(0)=0$ for $|\beta|<4$ and $|\beta|$ odd (see (3.46) and Proposition 3) we have

$$
\nu(\xi)=\sum_{m=0}^{\infty} \sum_{|\beta|=4+2 m} \frac{1}{\beta !} \xi^{\beta} \partial_{\xi}^{\beta} \alpha_{1}^{1}(0)
$$

and, consequently

$$
(-\nu(\xi))^{n}=(-1)^{n}\left(\sum_{m=0}^{\infty} \sum_{|\beta|=4+2 m} \frac{1}{\beta !} \xi^{\beta} \partial_{\xi}^{\beta} \alpha_{1}^{1}(0)\right)^{n}=\sum_{m=0}^{\infty} \sum_{|\beta|=4 n+2 m} c_{\beta, n} \xi^{\beta},
$$

for suitable constants $c_{\beta, n}$ that will be computed in Section 5. This fact suggests the following approximation

$$
\nu_{\alpha, p}(x, t) \sim \frac{1}{(2 \pi)^{N}} \int_{B_{\delta}} \xi^{\alpha}\left\{1+\sum_{n=1}^{p} \frac{t^{n}}{n !} \sum_{m=0}^{a(n)} \sum_{|\beta|=4 n+2 m} c_{\beta, n} \xi^{\beta}\right\} e^{-\frac{q_{k \ell}}{a_{0}} \xi_{k} \xi_{\ell} t} e^{i x \cdot \xi} d \xi,
$$

where $a(n)$ is an index to be chosen for any $n=1, \ldots, p$. Thus, let us define

$$
\begin{aligned}
\omega_{\alpha, p}(x, t) & =\frac{1}{(2 \pi)^{N}} \int_{B_{\delta}} \xi^{\alpha}\left\{1+\sum_{n=1}^{p} \frac{t^{n}}{n !} \sum_{m=0}^{a(n)} \sum_{|\beta|=4 n+2 m} c_{\beta, n} \xi^{\beta}\right\} e^{-\frac{q_{k \ell}}{a_{0}} \xi_{k} \xi_{\ell} t} e^{i x \cdot \xi} d \xi \\
& =I_{\alpha}^{*}(x, t)+\sum_{n=1}^{p} \frac{t^{n}}{n !} \sum_{m=0}^{a(n)} \sum_{|\beta|=4 n+2 m} c_{\beta, n} I_{\beta+\alpha}^{*}(x, t),
\end{aligned}
$$

with $a(n)$ to be chosen later, and consider the difference

$$
\begin{aligned}
& \nu_{\alpha, p}(x, t)-\omega_{\alpha, p}(x, t)= \\
& \quad=\frac{1}{(2 \pi)^{N}} \sum_{n=1}^{p} \frac{t^{n}}{n !} \int_{B_{\delta}} \xi^{\alpha}\left[(-\nu(\xi))^{n}-\sum_{m=0}^{a(n)} \sum_{|\beta|=4 n+2 m} \xi^{\beta} c_{\beta, n}\right] e^{-\frac{q_{k \ell}}{a_{0}} \xi_{k} \xi_{\ell} t} e^{i x \cdot \xi} d \xi .
\end{aligned}
$$

Now, computing in (3.52) the Taylor expansion of order $(4 n+2 a(n))$ of $(-\nu(\xi))^{n}$, we obtain the existence of a positive constant $C_{a(n)}>0$ satisfying, for all $\xi \in B_{\delta}$,

$$
\left|(-\nu(\xi))^{n}-\sum_{m=0}^{a(n)} \sum_{|\beta|=4 n+2 m} \xi^{\beta} c_{\beta, n}\right| \leq C_{a(n)}|\xi|^{4 n+2 a(n)+2} .
$$


Hence,

$$
\begin{gathered}
\left\|\nu_{\alpha, p}(\cdot, t)-\omega_{\alpha, p}(\cdot, t)\right\|^{2} \leq \\
\leq \sum_{n=1}^{p} \frac{t^{2 n}}{(n !)^{2}} \int_{B_{\delta}} \frac{|\xi|^{2|\alpha|}}{(2 \pi)^{2 N}}\left|(-\nu(\xi))^{n}-\sum_{m=0}^{a(n)} \sum_{|\beta|=4 n+2 m} \xi^{\beta} c_{\beta, n}\right|^{2} e^{-2 \frac{q_{k \ell}}{a_{0}} \xi_{k} \xi_{\ell} t} d \xi \\
\leq \frac{1}{(2 \pi)^{2 N}} \sum_{n=1}^{p} \frac{t^{2 n}}{(n !)^{2}} C_{\nu}^{2} \int_{B_{\delta}}|\xi|^{2|\alpha|}|\xi|^{2(4 n+2 a(n)+2)} e^{-2 \frac{q_{k \ell} \xi_{0} \xi_{k} t}{a_{0}} d \xi} \\
\leq c \sum_{n=1}^{p} t^{-\frac{N+4 n+4+4 a(n)+2|\alpha|}{2}}, \text { as } t \rightarrow \infty .
\end{gathered}
$$

Finally, in order to obtain the same decay rate as in Lemma 9 we take into account (3.49), and choose $a(n)$ satisfying $(N+4 n+4+4 a(n)+2|\alpha| \geq 2|\alpha|+4 p+4+N)$, i.e.,

$$
a(n) \geq p-n .
$$

Recalling that $p$ satisfies (3.50), we choose

$$
p(\alpha)=\left[\frac{k-|\alpha|}{2}\right] \text { and } a(\alpha, n)=\left[\frac{k-|\alpha|}{2}\right]-n .
$$

This gives us that

$$
\left\|\nu_{\alpha, p}(\cdot, t)-I_{\alpha}^{*}(\cdot, t)-\sum_{n=1}^{p(\alpha)} \frac{t^{n}}{n !} \sum_{m=0}^{a(n, \alpha)} \sum_{|\beta|=4 n+2 m} c_{\beta, n} I_{\beta+\alpha}^{*}(\cdot, t)\right\| \leq c t^{-\frac{2 k+n+2}{4}},
$$

as $t \rightarrow \infty$, and, since (3.51) is satisfied, we conclude the proof of Lemma $10 \square$.

In the sequel, we are going to prove the main result of this section, i.e., we obtain the complete asymptotic expansion of the solution of (1.11) when $\rho \equiv 1$.

Proof of Theorem 1 with $\rho \equiv 1$.

Firstly, for $(x, t) \in \mathbb{R}^{N} \times \mathbb{R}^{+}$, we denote by

$$
H(x, t)=\sum_{|\alpha| \leq k} c_{\alpha}(x)\left[G_{\alpha}^{*}(x, t)+\sum_{n=1}^{p(\alpha)} \frac{t^{n}}{n !} \sum_{m=0}^{a(\alpha, n)} \sum_{|\beta|=4 n+2 m} c_{\beta, n} G_{\alpha+\beta}^{*}(x, t)\right],
$$

the asymptotic expansion presented in Theorem 1 , where $G_{\alpha}^{*}(x, t)$ was defined in (1.7).

From Lemma 2 and Lemma 3 it follows that

$$
\begin{aligned}
\|u(\cdot, t)-H(\cdot, t)\| & \leq\|u(\cdot, t)-I(\cdot, t)\|+\|I(\cdot, t)-H(\cdot, t)\| \\
& \leq c e^{-a t}+\|I(\cdot, t)-H(\cdot, t)\|,
\end{aligned}
$$


where $I(x, t)$ is defined in (3.36). Then, to conclude the proof it is enough to prove that

$$
\|I(\cdot, t)-H(\cdot, t)\| \leq c_{k} t^{-\frac{2 k+2+N}{4}} \text {, as } t \rightarrow \infty .
$$

In fact, from Lemma 7 we have

$$
\begin{aligned}
\|I(\cdot, t)-H(\cdot, t)\| & \leq\|I(\cdot, t)-J(\cdot, t)\|+\|J(\cdot, t)-H(\cdot, t)\| \\
& \leq c_{k} t^{-\frac{2 k+2+N}{4}}+\|J(\cdot, t)-H(\cdot, t)\|, \quad \text { as } t \rightarrow \infty,
\end{aligned}
$$

and recalling that

$$
J(x, t)=\sum_{|\alpha| \leq k}(2 \pi)^{N} d_{\alpha} J_{\alpha}(x, t),
$$

where both, $J(x, t)$ and $J_{\alpha}(x, t)$, were defined in (3.37) and (3.40), respectly, we obtain from Lemma 9 ,

$$
\begin{aligned}
\|J(\cdot, t)-H(\cdot, t)\| & =\left\|\sum_{|\alpha| \leq k}(2 \pi)^{N} d_{\alpha}\left\{J_{\alpha}(\cdot, t)-\sum_{|\gamma| \leq k-|\alpha|} \widetilde{d}_{\gamma}(\cdot) I_{\gamma+\alpha}(\cdot, t)\right\}\right\| \\
& \leq c_{k}^{\prime} t^{-\frac{2 k+2+N}{4}}, \quad \text { as } t \rightarrow \infty
\end{aligned}
$$

Thus, if we define the periodic functions as

$$
c_{\alpha}(x)=(2 \pi)^{N} \sum_{\gamma \leq \alpha} \widetilde{d}_{\gamma}(x) d_{\alpha-\gamma},
$$

thanks to (3.53) and (3.54), it follows that

$$
\|I(\cdot, t)-H(\cdot, t)\| \leq c^{\prime} t^{-\frac{2 k+2+N}{4}}+\left\|\sum_{|\alpha| \leq k} c_{\alpha}(\cdot) I_{\alpha}(\cdot, t)-H(\cdot, t)\right\|, \text { as } t \rightarrow \infty .
$$

Now, we are going to prove that the following holds:

$$
\left\|\sum_{|\alpha| \leq k} c_{\alpha}(\cdot) I_{\alpha}(\cdot, t)-H(\cdot, t)\right\| \leq c_{k} t^{-\frac{2 k+2+N}{4}}, \quad \text { as } t \rightarrow \infty .
$$

Using Lemma 10, we obtain, as $t \rightarrow \infty$,

$\left\|\sum_{|\alpha| \leq k} c_{\alpha}(\cdot)\left[I_{\alpha}(\cdot, t)-I_{\alpha}^{*}(\cdot, t)-\sum_{n=1}^{p(\alpha)} \frac{t^{n}}{n !} \sum_{m=0}^{a(\alpha, n)} \sum_{|\beta|=4 n+2 m} c_{\beta, n} I_{\alpha+\beta}^{*}(\cdot, t)\right]\right\| \leq C_{k} t^{-\frac{2 k+2+N}{4}}$,

where $C_{k}$ depends on the $L^{\infty}$-norm of the functions $c_{\alpha}(\cdot)$ and the function $I_{\alpha}^{*}(x, t)$ defined in (3.44). Finally, Remark 8 gives us that

$$
\left\|\sum_{|\alpha| \leq k} c_{\alpha}(\cdot)\left(I_{\alpha}^{*}(\cdot, t)+\sum_{n=1}^{p(\alpha)} \frac{t^{n}}{n !} \sum_{m=0}^{a(\alpha, n)} \sum_{|\beta|=4 n+2 m} c_{\beta, n} I_{\alpha+\beta}^{*}(\cdot, t)\right)-H(\cdot, t)\right\| \leq c e^{-c(\delta) t},
$$


as $t \rightarrow \infty$, and, returning to $(3.53)$ we obtain

$$
\left\|\sum_{|\alpha| \leq k} c_{\alpha}(\cdot) I_{\alpha}(\cdot, t)-H(\cdot, t)\right\| \leq C_{k} t^{-\frac{2 k+2+N}{4}}+c e^{-c(\delta) t}, \quad \text { as } t \rightarrow \infty
$$

what concludes the proof $\square$.

\section{Proof of the general case}

Theorem 1 is proved following the same steps of Section 2 and 3 for the case $\rho \equiv 1$. However, the Bloch wave decomposition used for the equation (1.11) in Section 2 can not be applied for the problem (1.1), due to variable density $\rho$. Consequently, we need to introduce a different spectral problem.

Given $\xi \in Y^{\prime}$ we consider the spectral problem of finding numbers $\lambda=\lambda(\xi) \in \boldsymbol{R}$ and functions $\psi=\psi(x ; \xi)$ (no identically zero) such that

$$
\left\{\begin{array}{l}
A \psi(\cdot ; \xi)=\lambda(\xi) \psi(\cdot ; \xi) \rho(\cdot) \quad \text { in } \boldsymbol{R}^{N} \\
\psi(\cdot ; \xi) \text { is }(\xi, Y) \text {-periodic, i.e. } \\
\psi(y+2 \pi m ; \xi)=e^{2 \pi i m \cdot \xi} \psi(y) \quad \forall m \in Z^{N}, y \in \mathbb{R}^{N}
\end{array}\right.
$$

where $A$ is the elliptic operator in divergence form defined in (2.13) and $\rho$ satisfies (1.3). If we consider $\psi(x ; \xi)=e^{i x \cdot \xi} \phi(x ; \xi)$, the variational formulation obtained for (4.56) for any $\varphi \in H_{\#}^{1}(Y)$ is given by

$$
\langle A(\xi) \phi, \varphi\rangle=\int_{Y} a_{k \ell}(x)\left(\frac{\partial \phi}{\partial x_{k}}+i \xi_{k} \phi\right) \overline{\left(\frac{\partial \phi}{\partial x_{\ell}}+i \xi_{\ell} \phi\right)} d x=\lambda(\xi) \int_{Y} \phi \bar{\varphi} \rho(x) d x
$$

Since the operator associated with (4.56) is uniformly elliptic and self-adjoint, defined in a bounded domain, it is known ( see $^{3}$ and ${ }^{4}$ ) that the above spectral problem admits a discrete sequence of eigenvalues with the following properties:

$$
\left\{\begin{array}{l}
0 \leq \lambda_{1}(\xi) \leq \cdots \leq \lambda_{m}(\xi) \leq \cdots \rightarrow \infty \\
\lambda_{m}(\xi) \text { is a Lipschitz function of } \xi \in Y^{\prime}, \forall m \geq 1
\end{array}\right.
$$

Besides, the corresponding eigenfunctions denoted by $\psi_{m}(\cdot ; \xi)=e^{i \xi \cdot x} \phi_{m}(\cdot ; \xi)$, where the functions $\left\{\phi_{m}(x ; \xi)\right\}$ form orthonormal basis in the space of periodic functions in $L_{l o c}^{2}\left(\mathbb{R}^{N} ; \rho(x) d x\right)$, i.e,

$$
\int_{Y} \phi_{m} \bar{\phi}_{n} \rho(x) d x=\delta_{m n} \quad(\text { Kronecker's delta })
$$

The eigenfunctions $\psi_{m}(\cdot, \xi)$ and $\phi_{m}(\cdot, \xi)$ are $(\xi, Y)$-periodic and $Y$-periodic, respectively. Moreover, as a consequence of the min-max principle ( see $^{4}$ ) we have

$$
\lambda_{2}(\xi) \geq \frac{\lambda_{2}^{(N)}}{\rho_{1}}>0, \quad \forall \xi \in Y^{\prime},
$$


where $\lambda_{2}^{(N)}$ is the second eigenvalue of $A$ in the cell $Y$ with Neumann boundary condition on $\partial Y$ for $\rho \equiv 1$ and $\rho_{1}$ is defined in (1.3).

Now, with the orthonormal basis of Bloch waves $\left\{e^{i x \cdot \xi} \phi_{m}(x ; \xi): m \geq 1, \xi \in Y^{\prime}\right\}$, we have a similar Bloch wave decomposition as in Proposition 1:

Proposition 4 Let $g \in L^{2}\left(\mathbb{R}^{N}\right)$. The $m^{\text {th }}$ Bloch coefficient of $g$ is defined as follows:

$$
\widehat{g}_{m}(\xi)=\int_{R^{N}} g(x) e^{-i x \cdot \xi} \bar{\phi}_{m}(x ; \xi) \rho(x) d x \quad \forall m \geq 1, \xi \in Y^{\prime} .
$$

Then the following inverse formula holds:

$$
g(x)=\int_{Y^{\prime}} \sum_{m=1}^{\infty} \widehat{g}_{m}(\xi) e^{i x \cdot \xi} \phi_{m}(x ; \xi) d \xi
$$

Further, we have Parseval's identity:

$$
\|g\|_{L^{2}(\rho)}^{2}=\int_{R^{N}}|g(x)|^{2} \rho(x) d x=\int_{Y^{\prime}} \sum_{m=1}^{\infty}\left|\widehat{g}_{m}(\xi)\right|^{2} d \xi .
$$

Finally, for all $g$ in the domain of $A$, we have

$$
A g(x)=\rho(x) \int_{Y^{\prime}} \sum_{m=1}^{\infty} \lambda_{m}(\xi) \widehat{g}_{m}(\xi) e^{i x \cdot \xi} \phi_{m}(x ; \xi) d \xi .
$$

Using Proposition 4, the equation (1.1) can be written as follows:

$$
\int_{Y^{\prime}} \sum_{m=1}^{\infty}\left(\partial_{t}^{2} \widehat{u}_{m}(\xi, t)+\lambda_{m}(\xi) \widehat{u}_{m}(\xi, t)+a_{0} \partial_{t} \widehat{u}_{m}(\xi, t)\right) e^{i x \cdot \xi} \phi_{m}(x ; \xi) \rho(x) d \xi=0 .
$$

Since $\left\{e^{i x \cdot \xi} \phi_{m}(x ; \xi): m \geq 1, \xi \in Y^{\prime}\right\}$ form an orthonormal basis, this is equivalent to the family of the differential equations

$$
\partial_{t}^{2} \widehat{u}_{m}(\xi, t)+\lambda_{m}(\xi) \widehat{u}_{m}(\xi, t)+a_{0} \partial_{t} \widehat{u}_{m}(\xi, t)=0, \quad \forall m \geq 1, \xi \in Y^{\prime} .
$$

Once these differential equations are solved, (1.1) is solved as in (2.16) and Lemma 1 holds. The developments of Section 3 apply with minor changes and Theorem 1 holds.

In order to understand the type of changes that the variable density $\rho(\cdot)$ causes in the fundamental solution, we are going to study the Taylor expansion of the first Bloch eigenvalue and eigenvector. For a more complete analysis the reader is referred to ${ }^{2}$. We observe that

$$
\lambda_{1}(0)=0 \quad \text { and } \quad \phi(x ; 0)=(2 \pi)^{-\frac{N}{2}} \bar{\rho}^{-\frac{1}{2}},
$$


where $\bar{\rho}$ is defined in (1.8). We consider the equation

$$
A(\xi) \phi_{1}(\cdot ; \xi)=\lambda_{1}(\xi) \rho(\cdot) \phi_{1}(\cdot ; \xi)
$$

where

$$
A(\xi)=-\left(\frac{\partial}{\partial x_{k}}+i \xi_{k}\right)\left[a_{k \ell}(x)\left(\frac{\partial}{\partial x_{\ell}}+i \xi_{\ell}\right)\right] .
$$

If we differentiate the equation (4.59) with respect to $\xi_{k}$ with $k=1, \ldots, N$ and if we take scalar product with $\phi_{1}(x ; \xi)$ in $\xi=0$, we get

$$
\partial_{k} \lambda_{1}(0)=0 .
$$

Futhermore, we observe that

$$
A \partial_{k} \phi_{1}(\cdot ; 0)=i(2 \pi)^{-\frac{N}{2}} \bar{\rho}^{-\frac{1}{2}} \frac{\partial a_{k \ell}}{\partial x_{\ell}},
$$

then

$$
\partial_{k} \phi_{1}(x ; 0)=i(2 \pi)^{-\frac{N}{2}} \bar{\rho}^{-\frac{1}{2}} \chi^{k}(x),
$$

where $\chi^{k}$ is the classical test function in homogenization theory, solution of the cell problem

$$
\left\{\begin{array}{l}
A \chi^{k}=\frac{\partial a_{k \ell}}{\partial y_{\ell}} \quad \text { in } \quad Y \\
\chi^{k} \in H_{\#}^{1}(Y), \quad \frac{1}{|Y|} \int_{Y} \chi^{k} d y=0 .
\end{array}\right.
$$

This is the same test function as in the case $\rho \equiv 1$. If we differentiate again the eigenvalue equation, we have that

$$
\partial_{k \ell}^{2} \lambda_{1}(0)=\frac{1}{\bar{\rho}} \frac{1}{(2 \pi)^{N}} \int_{Y}\left(2 a_{k \ell}+a_{k m} \frac{\partial \chi^{\ell}}{\partial x_{m}}+a_{m \ell} \frac{\partial \chi^{k}}{\partial x_{m}}\right) d x=\frac{2 q_{k \ell}}{\bar{\rho}}
$$

with $q_{k \ell}$ the homogenized coefficients as in previous section (see in ${ }^{4}$ ).

Since $\alpha_{1}^{1}(\xi)$ is defined in (2.17) and thanks to the analysis above for the eigenvalue $\lambda_{1}$, we obtain

$$
\begin{array}{rl}
\alpha_{1}^{1}(0)=\partial_{k} \alpha_{1}^{1}(0)=0 & k=1, \cdots, N \\
\partial_{k \ell}^{2} \alpha_{1}^{1}(0)=\frac{2 q_{k \ell}}{\bar{\rho} a_{0}} & k, \ell=1, \cdots, N .
\end{array}
$$

Then, for all $\xi \in B_{\delta}$ we have

$$
e^{-\alpha_{1}^{1}(\xi) t} \sim e^{-\frac{q_{k \ell}}{\bar{\rho} \xi_{0}} \xi_{k} \xi_{\ell} t}
$$

\section{Analysis of the periodic functions and constants entering in the asymptotic expansion}


To finish this work we describe the periodic functions $c_{\alpha}(\cdot)$ and constants $c_{\beta, n}$, where $\alpha, \beta \in(N \cup\{0\})^{N}$ and $n \geq 1$, that appear in the statement of Theorem 1 .

Computation of $c_{\alpha}(\cdot)$. According to (3.38), (3.41) and (3.55),

$$
c_{\alpha}(x)=\sum_{\beta \leq \alpha} \frac{(2 \pi)^{N}}{(\alpha-\beta) ! \beta !} \partial^{\alpha-\beta} \phi_{1}(x ; 0) \partial^{\beta} \beta_{1}^{1}(0),
$$

where, recalling the definition of $\beta_{1}^{1}(\xi)$ given in (2.19), we have

$$
\begin{array}{r}
\partial^{\beta} \beta_{1}^{1}(0)=\partial^{\beta} \widehat{\varphi}_{1}^{0}(0)+\sum_{0 \neq \gamma \leq \beta} \frac{a_{0}}{2} \partial^{\beta-\gamma} \widehat{\varphi}_{1}^{0}(0) \partial^{\gamma}\left(\left(a_{0}^{2}-4 \lambda_{1}(\xi)\right)^{-\frac{1}{2}}\right)(0) \\
+\sum_{\gamma \leq \beta} \partial^{\beta-\gamma} \widehat{\varphi}_{1}^{1}(0) \partial^{\gamma}\left(\left(a_{0}^{2}-4 \lambda_{1}(\xi)\right)^{-\frac{1}{2}}\right)(0)
\end{array}
$$

and for $j=0,1$ ( the first Bloch coefficients of the initial data)

$$
\partial^{\gamma} \widehat{\varphi}_{1}^{j}(0)=\int_{R^{N}} \varphi^{j}(x) \sum_{\alpha \leq \gamma}\left[(-i)^{|\gamma-\alpha|} x^{\gamma-\alpha} \partial^{\alpha} \phi_{1}(x ; 0)\right] d x .
$$

We observe that the higher order derivatives of $\lambda_{1}$ and $\phi_{1}$ in $\xi=0$ may be computed as in the previous section.

First, note that

$$
c_{0}(x)=(2 \pi)^{N} \phi_{1}(x ; 0) \beta_{1}^{1}(0)=(2 \pi)^{N} \phi_{1}(x, 0)\left(\widehat{\varphi}_{1}^{0}(0)+\frac{\widehat{\varphi}_{1}^{1}(0)}{a_{0}}\right),
$$

and since $\phi_{1}(x, 0)=(2 \pi)^{-\frac{N}{2}} \bar{\rho}^{-\frac{1}{2}}$, it follows that $c_{0}$ is constant. Futhermore, according to Proposition 4, we have for $i=0,1$

$$
\widehat{\varphi}_{1}^{i}(0)=(2 \pi)^{-\frac{N}{2}} \bar{\rho}^{-\frac{1}{2}} \int_{R^{N}} \varphi^{i}(x) \rho(x) d x=(2 \pi)^{-\frac{N}{2}} \bar{\rho}^{-\frac{1}{2}} m_{\rho}\left(\varphi^{i}\right) .
$$

Thus,

$$
c_{0}:=c_{0}(x)=\frac{1}{\bar{\rho}} m_{\rho}\left(\varphi^{0}+\frac{1}{a_{0}} \varphi^{1}\right),
$$

and, since (1.4) is satisfied, $c_{0}$ is defined as in (1.10).

For $|\alpha|=1$, we consider $\alpha=e_{k}$, any of the canonical vectors. The corresponding periodic function is

$$
c_{k}(x)=\frac{-i}{\bar{\rho}}\left(\chi^{k}(x) m_{\rho}\left(\varphi^{0}+\frac{\varphi^{1}}{a_{0}}\right)+m_{\rho}\left(\left(\chi^{k}+x_{k}\right)\left(\varphi^{0}+\frac{\varphi^{1}}{a_{0}}\right)\right),\right.
$$

with $\chi^{k}$ the periodic test function, solution of (4.60). Observe that two different terms appear in $c_{k}$. First the total mass of the solution multiplied by the periodic 
function $\chi^{k}(\cdot)$. Second, a constant term in which the periodic function $\chi^{k}(\cdot)$ enters as well, but this time as a weight when computing the corresponding moment of the initial data. In both cases we see how the periodicity of the medium affects the value of $c_{k}$ that varies substantially with respect to the case of constant coefficients. This fact was already pointed out in ${ }^{7}$ when studying the heat equation with periodic coeffcients.

The remaining values of the functions $c_{\alpha}(\cdot)$ with multiindexes $\alpha$ with $|\alpha| \geq 2$ may be computed by taking successive derivatives in (4.59).

Computation of $c_{\beta, n}$. We recall that the constants $c_{\beta, n}$ were defined in (3.52) and satisfy

$$
\sum_{m=1}^{\infty} \sum_{|\beta|=4 n+2 m} \xi^{\beta} c_{\beta, n}=(-1)^{n}\left(\sum_{m=0}^{\infty} \sum_{|\beta|=4+2 m} \frac{1}{\beta !} \xi^{\beta} \partial^{\beta} \alpha_{1}^{1}(0)\right)^{n},
$$

where $\alpha_{1}^{1}(\xi)$ is given in (2.17). Moreover, we have for $|\beta|$ even and $|\beta| \geq 4$ that

$$
\partial^{\beta} \alpha_{1}^{1}(0)=\sum_{m=1}^{|\beta|} \sum_{\substack{\beta^{\prime}, \beta_{1}, \ldots, \beta_{N} \in \in_{N} \\ \beta^{\prime}+\beta_{1}+\cdots+\beta_{N}=\beta}}^{\left|\beta^{\prime}\right|=m} \frac{2^{m-1} f(m)}{a_{0}^{2 m-1}} \partial^{\beta_{1}}\left[\left(\partial_{1} \lambda_{1}\right)^{\beta_{1}^{\prime}}\right](0) \cdots \partial^{\beta_{m}}\left[\left(\partial_{N} \lambda_{1}\right)^{\beta_{N}^{\prime}}\right](0),
$$

where, for $m \in N$,

$$
f(m)=\left\{\begin{array}{cl}
1 & m=1, \\
\frac{(2 m-3) !}{2^{m-2}(m-2) !} & m \geq 2 .
\end{array}\right.
$$

Observe that the constants $\partial^{\beta} \alpha_{1}^{1}(0)$ depend on the derivatives of $\lambda_{1}$ at $\xi=0$, computed in Section 4. Thus, we may write the constants $c_{\beta, n}$ as

$$
c_{\beta, n}=\sum_{\substack{s_{1}, \ldots, s_{n} \in \mathbb{N} \\ s_{1}+\cdots+s_{n}=\frac{|\beta|-4 n}{2}}} \sum_{\substack{\beta_{1}, \ldots, \beta_{n} \in \mathbb{N}^{N} \\ \beta_{1}+\cdots+\beta_{n}=\beta}}^{\left|\beta_{i}\right|=4+s_{i}}(-1)^{n} \frac{1}{\beta_{1} !} \cdots \frac{1}{\beta_{n} !} \partial^{\beta_{1}} \alpha_{1}^{1}(0) \cdots \partial^{\beta_{n}} \alpha_{1}^{1}(0) .
$$

\section{Acknowledgment}

This work was done while the second author was a visiting scholar at Universidad Complutense de Madrid in post-doctoral program supported by grant BEX1432/990 of the CAPES (MCT-BRASIL). The first and third authors were supported by grant PB96-0663 of the DGES (SPAIN) and the TMR project of the EU "Homogenization and Multiple Scales".

\section{References}

1. C. M. Bender and S. A. Orszag, Advanced mathematical methods for scientists and engineers, (McGraw-Hill, 1978). 
2. C. Conca, R. Orive, and M. Vanninathan, Bloch approximation in homogenization and applications, preprint (2000).

3. C. Conca, J. Planchard, and M. Vanninathan, Fluids and Periodic Structures, no. 38 in Research in Applied Mathematics, (J. Wiley/Masson, 1995).

4. C. Conca and M. Vanninathan, Homogenization of periodic structures via Bloch decomposition, SIAM J. Appl. Math. 57 (1997) 1639-1659.

5. J. Duoandikoetxea and E. Zuazua, Moments, masses de Dirac et décomposition de fonctions, C. R. Acad. Sci. Paris Sér. I Math. 315 (1992) 693-698.

6. G. Duro and E. Zuazua, Large time behavior for convection-diffusion equations in $\boldsymbol{R}^{N}$ with asymptotically constant diffusion Comm. Partial Differential Equations 24 (1999) $1283-1340$.

7. J. H. Ortega and E. Zuazua, Large time behavior in $\boldsymbol{R}^{N}$ for linear parabolic equations with periodic coefficients, Asymptotic Anal. 22 (2000) 51-85. 